\title{
Loose combined cutting seton for patients with suprasphincteric anal fistula: a randomized clinical trial protocol
}

\author{
Yicheng Cheng ${ }^{1}$, Lihua Zheng ${ }^{1}$, Yuying Shi ${ }^{1}$, Congcong Zhi ${ }^{1}$, Jiaying Shan ${ }^{2}$, Yaxuan Sun ${ }^{2}$, Hongxin Guo ${ }^{2}$, \\ Dun Liu', Yan Zhang' \\ ${ }^{1}$ Anorectal Department, China-Japan Friendship Hospital, Beijing, China; ${ }^{2}$ Graduate School, Beijing University of Chinese Medicine, Beijing, China \\ Correspondence to: Lihua Zheng. Anorectal Department, China-Japan Friendship Hospital, Beijing 100029, China. Email: btv1262021@163.com.
}

\begin{abstract}
Background: Suprasphincteric anal fistula is a type of high anal fistula. The traditional method of cutting seton (CS) has a high recurrence rate and can cause severe damage to the anal sphincter and anal incontinence. The combination of loose and cutting seton is a novel method developed on the basis of the traditional cutting seton technique, and has already been adopted by some clinicians in China. This study will examine the effectiveness and safety of the loose combined cutting seton (LCCS) technique for the treatment of suprasphincteric anal fistulas.
\end{abstract}

Methods: This is a single-blinded randomized controlled trial conducted in the Anorectal Department of the China-Japan Friendship Hospital. A total of 76 patients diagnosed with suprasphincteric anal fistula will be randomly divided into two groups. One group will be treated with the LCCS method (the LCCS group; $\mathrm{n}=38$ ) and the other group will be treated with the traditional CS method (the CS group; $n=38$ ). There will be 3 intervention periods, including the screening period, the surgical treatment period, and the postoperative follow-up period. Postoperative follow-up will be carried out on days 3, 5, 7, 14, 21, 28, 90, 180, and 365 after the operation. The main outcome measures are the complete cure rate of postoperative wounds and fistulas, the long-term recurrence rate, and evaluation of postoperative anal function (Wexner anal function assessment and anal function questionnaire). The secondary outcomes are the visual analogue scale (VAS) score for postoperative pain, pressure measurements of the anal canal and rectum before and after treatment, and the incidence of adverse events. All statistical results will be analyzed using the SPSS software 21.0 version. $P$ values $<0.05$ will be considered statistically significant.

Discussion: This research introduces a novel method for the treatment of suprasphincteric anal fistulas. The LCCS method will be compared with the traditional CS method in terms of safety and efficacy. If the LCCS technique is a safe and effective treatment for suprasphincteric anal fistula, its clinical application should be promoted.

Trial registration: ClinicalTrials, Registration number: ChiCTR2100045450; pre-results.

Protocol version: 2020-09-10 1.0 version.

Keywords: Suprasphincteric anal fistula; effectiveness; recurrence; anal function; loose combined cutting seton (LCCS)

Submitted Jul 19, 2021. Accepted for publication Sep 15, 2021.

doi: 10.21037/apm-21-2303

View this article at: https://dx.doi.org/10.21037/apm-21-2303

\section{Introduction}

\section{Background}

Anal fistula is an abnormal passage between the anorectal and perianal skin. It is a common anorectal disease, with an incidence rate second only to hemorrhoids. According to the latest epidemiological statistics, the foreign incidence of anal fistula in anorectal diseases is about $8 \%$, and domestically, it is $1.67-3.60 \%$. The peak age of onset is 20-40 years old, and tends to affect males more than 
females (1-5). Anal fistulas are mostly caused by anal gland infections. The clinical manifestations are induration of the anus, repeated local ulceration, pus, and pain, all of which significantly decrease the patient's quality of life. According to Parks classification, the incidence of suprasphincteric anal fistula accounts for about $4 \%$ of anal fistulas (6). A complicated anal fistula is formed when the fistula is complicated or accompanied by a branch duct and deep dead space, which can cause repeated perianal infections. The infection spreads along the muscle space and invades the pelvic cavity, which can cause serious complications such as rectovaginal fistula, rectourethral fistula, and rectovesical fistula. Other complications include infections such as sepsis and cancer (7-9)

Suprasphincteric anal fistulas are not self-resolving and must be treated surgically. The purpose of the operation is to remove the infected fistula and epithelialized fistula, while minimizing damage to anal function. The anorectal ring is the control center of the anus, and functions to maintain anal control and assist defecation. It is composed of the internal and external sphincter, the puborectalis, the levator ani muscle, and other muscle tissues. Suprasphincteric anal fistula is a high anal fistula and the infection lesion involves the anal straight ring. If the lesion is removed, it will lead to anal incontinence, and if the lesion is retained, there is a high risk of relapse, making suprasphincteric anal fistula a refractory disease. The associated severe pain, high recurrence rate, and postoperative anal incontinence, can cause severe mental and economic stress to patients.

To date, there is still no unified standard for the treatment of suprasphincteric anal fistulas. Therefore, exploring suitable methods to improve the success rate of suprasphincteric anal fistula treatment, reduce recurrence, avoid anal function damage, reduce pain, and improve the quality of life, is warranted.

The current treatments for suprasphincteric anal fistula mainly focus on sphincter preservation, and include ligation of intersphincteric fistula tract (LIFT), fibrin glue sealant (FGS), fistula plug, video-assisted anal fistula treatment (VAAFT), fistula-tract laser closure (FILAC), and over-thescope clip (OTSC) (10-16). For intersphincteric fistulas with short fistulas, LIFT is difficult to separate and ligate. Compared with anal fistula resection with high cure rates and fewer complications, LIFT has certain limitations. FGS is a treatment method in which fibrin glue is injected into the fistula after the internal opening and the fistula are scraped, and a clot is formed in contact with the wound surface, thereby filling the fistula. However, studies have shown that the cure rate of fibrin glue occlusion fluctuates greatly, ranging from $14 \%$ to $90 \%$ (17). Therefore, achieving high cure rates while protecting anal function remains a significant challenge.

Traditional Chinese medicine (TCM) administers cutting seton therapy to treat anal fistulas and this method is widely applied in China. There are two types of cutting seton techniques, namely, the actual seton method and the virtual seton method. At present, actual seton therapy is the firstline treatment for various types of anal fistulas. The actual seton method aims to effectively protect the function of the sphincter by gradually tightening the thread to achieve the purpose of slowly cutting the sphincter. This method is simple, results in little bleeding, and a low recurrence rate. However, the wound is large, the patient experiences considerable pain, and there is a lengthy wound healing time. While actual seton therapy can avoid the retraction of the sphincter, in practice, it is necessary to tighten the thread several times to ensure the effect, and this can easily lead to anal canal deformity, postoperative scars, and severe pain, and is further compounded by the risk of total or partial anal incontinence (18-21).

The virtual seton method promotes fibrosis by loosely applying the thread so as to achieve the therapeutic purpose of short-term and long-term drainage. The virtual seton is hooked with a rubber band for drainage, which helps to maintain the normal sphincter function of the anus. While this method has been clinically proven to relieve symptoms in the short-term, it is difficult to achieve long-term cure and the recurrence rate is high (22-24).

Based on these two cutting seton methods, the "loose combined cutting seton" (LCCS) technique will be applied in this study. This involves an actual seton operation on the suprasphincteric anal fistula and after the actual seton is disconnected from part of the sphincter, a virtual seton is formed. The virtual thread will be kept for about 21 days to ensure the healing process is successful. This therapy has been shown to have a definite curative effect in preliminary clinical observations, and postoperative follow-up observations have demonstrated that LCCS can preserve the function of the patient's anal sphincter with low incontinence rates and a $100 \%$ cure rates and no recurrence (25). A single-center randomized controlled trial will be conducted to observe and compare the clinical efficacy and the incidence of postoperative complications of LCCS and traditional CS therapy. This will enable clinicians make evidence-based decisions in the treatment of patient with suprasphincteric anal fistulas. 


\section{Study objectives}

The study will evaluate the clinical efficacy of the LCCS method compared to the traditional CS method in the treatment of suprasphincteric anal fistulas. The insights gained will provide a clinical basis for the treatment of patients with suprasphincteric anal fistulas. We present the following article in accordance with the SPIRIT reporting checklist (26) (available at https://dx.doi.org/10.21037/apm21-2303).

\section{Methods}

\section{Study design}

The study is a prospective, randomized controlled, singleblinded and single-center non-inferiority clinical trial.

\section{Research setting}

In this study, patients diagnosed with suprasphincteric anal fistula and who plan to be admitted for surgical treatment at the Anorectal Department of the China-Japan Friendship Hospital (Beijing, China) will be enrolled.

\section{Enrollment and eligibility criteria}

All patients diagnosed with suprasphincteric anal fistula can participate in this study. The diagnostic criteria of suprasphincteric anal fistula are based on the Parks classification of anal fistula in the American Society of Colorectal Surgeons (ASCRS) 2005 edition of the Anal Fistula Diagnosis and Treatment Guidelines.

\section{Inclusion criteria}

Subjects must meet all of the following inclusion criteria:

(I) Age $\geq 18$ years old and $\leq 65$ years old, male or female;

(II) Diagnosis of suprasphincteric anal fistula is confirmed with transrectal B-ultrasound, in accordance with the Parks diagnostic criteria;

(III) The patient will undergo anal surgery for the first time; and

(IV) The patient voluntarily participates in this clinical trial, signs the informed consent form, and can cooperate with clinical follow-up.

\section{Exclusion criteria}

Potential subjects will be excluded from this study if:

(I) The suprasphincteric anal fistula is caused by trauma;

(II) The patient presents with acute infection or poorly controlled lesion infection;

(III) The patient presents with other medical history that affects anal function;

(IV) The patient cannot receive this treatment due to religious or ethnicity reasons, diabetes, cancer, intestinal tuberculosis, Crohn disease, or AIDS;

(V) The patient presents with comorbidities such as severe heart, lung, brain, liver, or kidney disease;

(VI) The patient is pregnant or planning pregnancy, or is lactating;

(VII) The patient presents with allergic constitution or multiple drug allergies.

\section{Intervention}

The research process is divided into three periods (Table 1).

The first period is the screening phase and involves recruiting participants according to the inclusion and exclusion criteria and obtaining signed informed consent forms prior to inclusion. Before enrollment, the patient's medical history will be obtained, and the necessary screening examinations, such as digital rectal examination and intrarectal ultrasound, will be performed.

The second period is the treatment phase, which includes preoperative preparation, surgery, and postoperative management. All patients will undergo the same preoperative preparation including anorectal cavity color Doppler ultrasound examination, exclusion of contraindications for surgery, fasting from water and food for 8 hours, emptying the bowels, and administering an enema if required. In order to minimize the bias between surgeons, we ensure that all procedure will be conducted by the same experienced doctor.

For the surgery, patients will be randomly assigned into the LCCS group or the CS group. All patients will be placed in a lateral position, routinely disinfected, draped, and placed under intravenous anesthesia. After the anal canal becomes loose, it is disinfected and a digital anal examination will be performed to determine the area and location of the anal fistula, and whether there are branches and dead spaces. During the LCCS operation, a radial incision is made from the inner mouth outward from the dentinal line, about 3 to $4 \mathrm{~cm}$ in length, and the incision position is generally on the same side as the outer orifice to fully drain the infection foci at the inner mouth. The inner orifice is cut, extending $0.5-1.0 \mathrm{~cm}$ upwards, and extending 
Table 1 Timeline of study

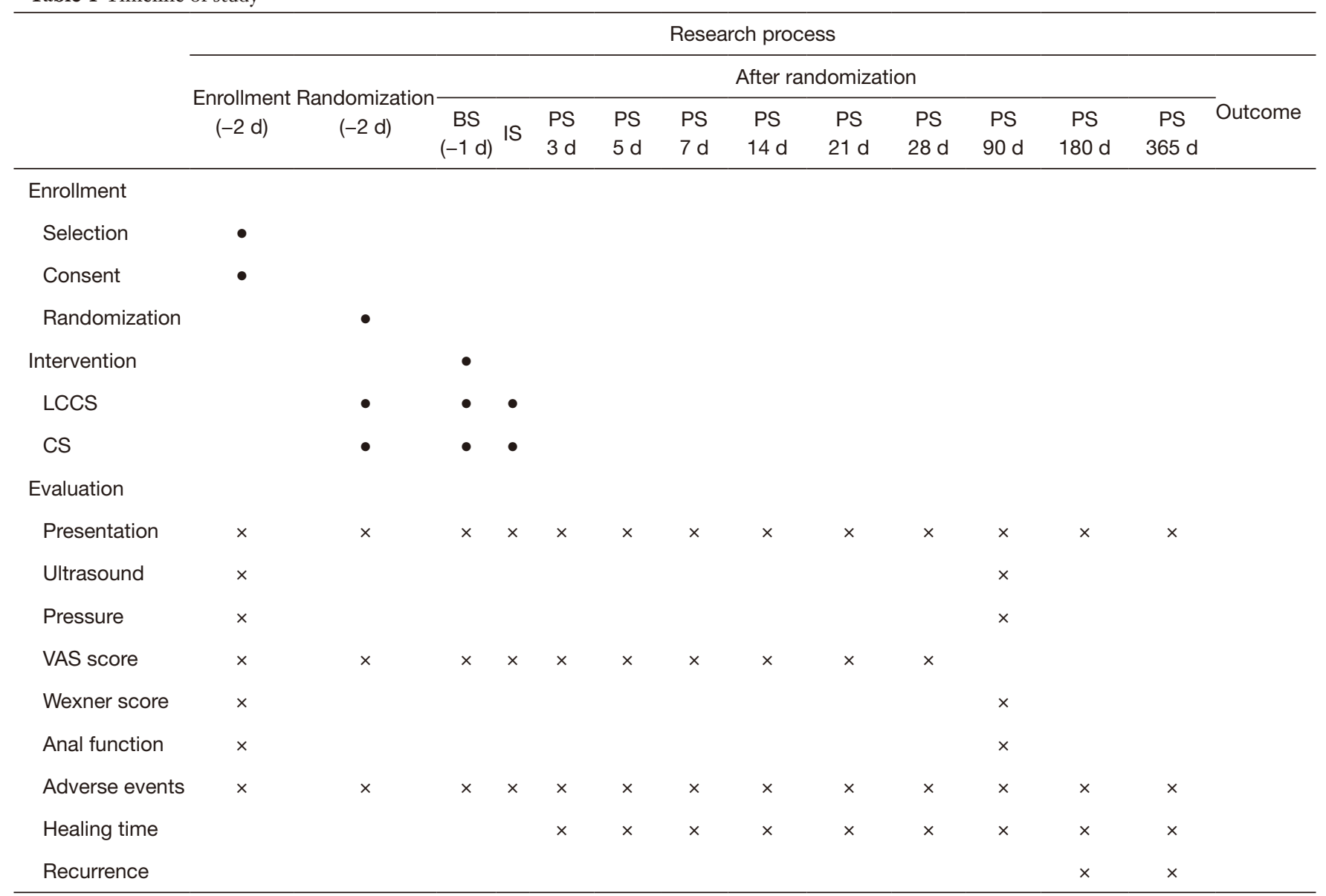

BS, before surgery; IS, intro-surgery; PS, post-surgery; LCCS, loose combined cutting seton; CS, cutting seton; VAS, visual analogue scale. $\bullet$ represents done by team leader; $x$ represents done by team members.

downwards to the outside of the anal margin. The curved hemostatic forceps is used to probe the upper end of the fistula from this incision until the top of the fistula. One finger is used to penetrate the intestinal cavity for guidance, and the tip of the forceps is used to penetrate the stoma of the intestinal wall. The finger is withdrawn and using four 10 -gauge silk threads, one end is tied to the fingertip and placed into the intestinal cavity. The hemostatic forceps is opened to clamp the thread, and the silk thread is drawn from the intestinal cavity through the fistula. The two ends are gathered and fixed with a knot. Routine cleaning and dressing changes will be performed daily post-operation. The Vaseline gauze will be drained and replaced. At around 7 days after the operation, the hanging silk thread will be loose and at this time, the virtual thread will be drained. After the granulation tissue of the fistula is filled, the thread will be removed on postoperative day 20 .
During the CS operation, the internal port is located, the top of the fistula is placed in the intestinal cavity, and a rubber strip is inserted from the internal port and pulled out from the top stoma. Both ends are tightened forcefully and tied together with a silk thread. The thread will be tightened again according to the elasticity of the rubber band at approximately 7 days after the operation. Generally, the thread must be tightened repeatedly 3-4 times until the fistula is completely cut. If there is an external orifice outside the anal margin, the lower fistula should be treated with incision.

Both groups of patients will receive postoperative management which includes routine intravenous infusion of broad-spectrum antibiotics and anti-anaerobic antibiotics for 3 days after surgery. Patients who have bowel movements within 24 hours post-operation will have their dressing changed immediately. For patients who pass stool more than 24 hours post-operation, the dressing will be changed at 


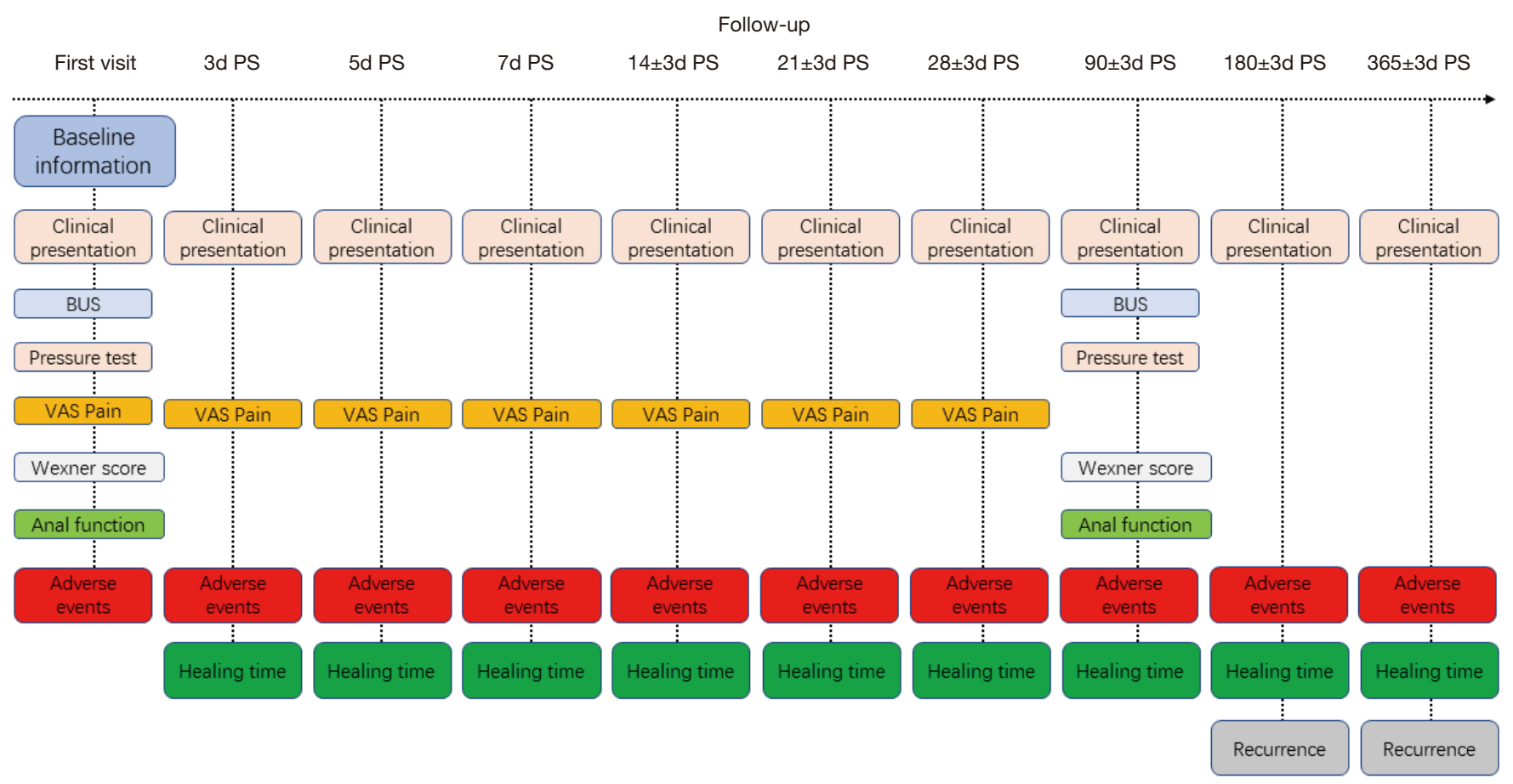

Figure 1 Follow-up timepoints and assessments.

24 hours after the operation. Dressings will be changed twice a day. Before each dressing change, patients will be treated with traditional Chinese medicine preparations to fumigate and bathe for 10 minutes. The anorectal fumigation and washing preparation contains gallnut $15 \mathrm{~g}$, dandelion $30 \mathrm{~g}$, raw Platycladus orientalis $15 \mathrm{~g}$, Sophora flavescens 30 g, Glauber's salt 15 g, Cangzhu 15 g, Sanyu 15 g, Fangfeng $15 \mathrm{~g}$, Phellodendron amurense $30 \mathrm{~g}$, Red peony root $15 \mathrm{~g}$, Honeysuckle $15 \mathrm{~g}$, and raw licorice $10 \mathrm{~g}$. The preparation is wrapped in gauze, put it in a basin, and brewed with 2,000 $\mathrm{mL}$ hot water. When the solution reaches about $40^{\circ} \mathrm{C}$, the anus and wound is washed for 10 minutes.

The third period is the follow-up phase which lasts for at least 1 year and includes 10 medical visits. Telephone follow-up or face-to-face follow-up consultations will be conducted on postoperative days $3,5,7,14,21,28,90,180$, and 365 . At each follow-up, the patient's current clinical presentation will be assessed. Since the longest time limit for the postoperative recovery period is 3 months, anorectal ultrasound, evaluation of rectal pressure, anal function score, and anal Wexner incontinence score will be assessed at this time. At other follow-up timepoints, the patient will self-report symptoms and recurrence. If necessary, the patient's anal function will be reassessed at the 6-month and 1-year follow-up. During clinical observations, it has been noted that some patients may experience transient anal incontinence due to the effects of postoperative scars. Therefore, addition assessments of anal function will provide a clearer picture of the patient's postoperative anal recovery. The specific follow-up timepoints and assessments are shown in Figure 1.

\section{Outcome measures}

Outcomes were assessed in terms of efficacy and safety. The main efficacy evaluation index is the complete cure rate of postoperative wounds and fistulas. The secondary efficacy evaluation indicators include symptoms and signs before and after surgery, such as swelling and pain, and discharge of pus; recurrence in six months and one year; and anorectal ultrasound. Data will be collated and differences in the 2 treatment groups will be compared in terms of recovery rate, effective rate, inefficiency, average healing time, and recurrence rate. Anorectal B-ultrasound will be used as an objective evaluation index to assess the treatment of suprasphincteric anal fistula with the LCCS. The safety observation indicators include postoperative pain visual analogue scale (VAS) score; anal canal and 
rectal pressure measurements before and after treatment; evaluation of anal function before and after treatment including the Wexner anal function assessment and the anal function questionnaire; and the incidence of adverse events. The differences between the two groups in terms of postoperative pain, anal pressure measurement, and anal function will be compared.

\section{Primary outcomes}

The main curative effect evaluation index is complete cure rate of postoperative wounds and fistulas. Data will be collated and differences in the 2 treatment groups will be compared in terms of recovery rate, effective rate, inefficiency, average healing time, and recurrence rate. Anorectal B-ultrasound will be used as an objective evaluation index to assess the treatment of suprasphincteric anal fistula with the LCCS.

\section{Secondary outcomes}

Secondary outcomes include both efficacy evaluation indicators and safety evaluation indicators. The secondary efficacy evaluation indicators include symptoms and signs before and after surgery such as swelling and pain and discharge of pus; half-year and one-year recurrence rates; and anorectal B-ultrasounds. Safety evaluation indicators include VAS score for postoperative pain, measurement of anal and rectal pressure before and after treatment, assessment of anal function before and after treatment by Wexner anal function assessment, and incidence of adverse events.

\section{Sample size}

This study is a non-inferiority clinical randomized controlled trial. The cure rate is the main outcome indicator. $\mathrm{N}$ is the sample size. The ratio of the LCCS group and the control CS group is 1:1. According to previous literature reports and clinical application summary, the cure rate of the CS group and the LCCS group is set to $95 \%$, that is $\mathrm{P} 2=0.95$. the test level ais 0.025 (single-sided). Test power is 0.80 . The noninferiority threshold is $\mathrm{d}=0.15$. Using the PASS15 sample size software, the sample size $\mathrm{N}_{1}=34$ of the LCCS group was obtained, and the sample size of the CS group was $\mathrm{N}_{2}=34$ cases. Assuming that the loss to follow-up rate of the research subjects is $10 \%$, the sample size is $\mathrm{N}_{1}=34 \div 0.9=38$ cases, and $\mathrm{N}_{2}=34 \div 0.9=38$ cases.

\section{Recruitment}

Recruitment for this trial will be carried out through the internet and advertisements within clinics. After registration, the subjects will be enrolled if they pass all regular medical treatment procedures and screening according to the inclusion and exclusion criteria.

\section{Method of allocation of interventions}

\section{Randomization and blinding}

This study uses a randomized, controlled, single-blinded research method. Before enrollment, patients will be informed that the surgeon will use a method with definite curative effect to perform the operation, which may be a traditional method or a relatively novel method. The specific operation method will be determined by the surgeon. In order to minimize the bias of the trial, after the clinical follow-up is completed, the data will be sent to a third party for statistical analysis. The third party is blinded to which set of data belongs to the experimental group and which set belongs to the control group. Finally, the physician and the statistical analysis party jointly announce the corresponding grouping of the results.

\section{Random method}

The assigned serial number is generated by the anorectal doctor in the China-Japan Friendship Hospital using SAS software. According to the block random table generated by the SAS software, the random sequence is formed and divided into 2 groups according to the random sequence number. The larger number is the LCCS group. The smaller number is the CS group. The two sets of random sequences are then placed into sealed opaque envelopes and arranged in random sequence. The envelopes are kept by a nurse who is completely blinded to the content of this study. At the time of allocation, the selected cases will receive a random serial numbers and the corresponding treatment methods will be carried out.

\section{Statistical analysis}

The SPSS 22.0 statistical software package will be used for statistical analysis. All statistical tests performed will be two-sided tests, and a $\mathrm{P}$ value of $<0.05$ will be considered statistically significant.

Data will be presented as mean and standard deviation or median and interquartile range for blood pressure, heart rate, 
body temperature, anorectal pressure, and wound and fistula final healing time. The number of cases and the percentage of classification indicators such as gender, wound pain degree score, anal function evaluation score, and curative effect evaluation results will be described. The Kaplan Meier method will be used to estimate the healing rate at each timepoint after surgery, and the log rank test method will be used to compare the healing time between the two groups.

The mean \pm standard deviation will be used to describe the resting pressure of the anorectal anal canal, the length of the anal hypertension zone, and the maximum systolic pressure of the anal canal before treatment and after healing in the LCCS group and the CS group. The above indicators before treatment will be used as covariates and the analysis of covariance will be used to compare the difference between the test group and the control group.

Quantitative data will be compared between the two groups. For example, when comparing the final healing time of wounds and fistulas between the LCCS group and the CS group, normality and homogeneity of variance will be considered. If normality and uniform variance are satisfied, the t-test will be used. For non-conformity data, the Wilcoxon rank sum test will be applied. Comparison of qualitative data between the two groups, such as the results of anal function curative effect evaluation, will be performed using the $\chi^{2}$ test and the rank sum test will be used for grade data. The paired signed-rank test will be used to compare the changes of incontinence scores before and after treatment in each group, and the one-way ordered Cochran-Mantel-Haenszel (CMH) $\chi^{2}$ test will be used to compare between groups.

\section{Record of adverse events}

All adverse events and serious adverse events occurring during the clinical trial will be documented. If a patient or clinical trial subject experiences an adverse medical event after receiving treatment, but it is not necessarily causally related to the treatment, it is called an adverse event. Serious adverse events are defined as events that require hospitalization or prolong hospitalization, cause disability or affect work ability, are life-threatening, or cause death or congenital malformations. Clinical adverse events may occur during the treatment of subjects. Once adverse events (including important adverse events) occur, the time of occurrence, clinical manifestations, treatment process and duration, outcome, the relationship with the drug and related events should be recorded in detail on the case report form. If there is an abnormal laboratory test, the patient must be followed up until the test result returns to normal, or to the level before the drug administration, or it is determined that it unrelated to the drug. Serious adverse events should be documented using the serious adverse event form and reported to the ethics committee within 24 hours.

\section{Ethics committee review}

This protocol, written informed consent forms, and materials directly related to the subjects must be submitted to the ethics committee. The research can only be formally commenced after obtaining written approval from the ethics committee. The investigator must submit an annual research report to the ethics committee (if applicable). When the research is suspended and/or completed, the researcher must notify the ethics committee in writing. The researcher must report to the ethics committee all changes to the research work (such as the revision of the protocol and/or the number of informed consents). These changes may not be implemented until approved by the committee unless they are made to eliminate obvious and direct risks to the subject. In such cases, the ethics committee will be notified.

\section{Informed consent}

\section{Procedure for obtaining informed consent}

The researcher must provide the subject or his legal representative with an easy-to-understand informed consent form approved by the ethics committee, and give the subject or his legal representative sufficient time to consider this research. Subjects are not allowed to enter the group before providing signed written informed consent forms. During the participant's participation, all updated informed consent forms and written information will be provided to the participants. The informed consent form should be kept as an important document for clinical trials for future reference.

\section{Confidentiality measures}

The results of the research through this project may be published in medical journals. All patient information will be kept confidential in accordance with the requirements of the law, and will not be disclosed unless required by relevant laws. When necessary, government management departments, hospital ethics committees and their related personnel can consult the patient's data in accordance with regulations. 


\section{Ethics and dissemination}

This study was approved by the Institution Review Board of the China-Japan Friendship Hospital (Approval number: 2020-89-K53). The results of this study will be submitted to international scientific peer-reviewed journals or conferences in surgery, anorectal surgery, or anorectal diseases. All procedures performed in this study involving human participants will be in accordance with the Declaration of Helsinki (as revised in 2013). Informed consent will be obtained from each patient before taking part.

\section{Discussion}

To date, there is no consensus on the surgical treatment of anal fistulas. Complex anal fistulas are complicated by high risk of treatment failure and inability to be treated by conventional fistula incisions. In addition, the risk of postoperative incontinence with only one-stage fistula incision is high, and anal preservation procedures are required, such as intraanal mucosal flap replacement, fibrin glue, anal fistula plug, modified Hanley surgery, LIFT, and diversion surgery. The choice of surgical method depends on the type of fistula and the doctor's preference. Among many surgical methods, the thread hanging method is the more recognized and more readily applied both in China and overseas. However, there are significant limitations to this method, for example, the drainage thread may not cut the sphincter. In high anal fistula surgery, the drainage thread can be used to protect the function of the sphincter and help eradicate sepsis (27), but this has little effect on the fistula. The cutting seton uses reactive suture or elastic threads to pass through the fistula which is then tightened regularly. This can slowly cut the fistula, creating scars, thereby preventing extensive damage to the anal sphincter by fistula incision. But the pain of the cutting is obvious, and the patient has a greater risk of incontinence (28). LCCS can reduce the pain experienced by patients and the manpower required by doctors to repeatedly tighten the thread to the greatest extent. This method has been preliminarily proven to have a high healing rate and good safety (25). The purpose of this single-center randomized controlled trial is to further verify the efficacy and safety of the LCCS method, in order to promote its clinical applications.

\section{Acknowledgments}

Funding: This study was funded by the Wu Jieping Medical
Foundation (Project number: 320.6750.2020-8-34).

\section{Footnote}

Reporting Checklist: The authors have completed the SPIRIT reporting checklist. Available at https://dx.doi. org/10.21037/apm-21-2303

Conflicts of Interest: All authors have completed the ICMJE uniform disclosure form (available at https://dx.doi. org/10.21037/apm-21-2303). All authors report funding support from the Wu Jieping Medical Foundation (Project number: 320.6750.2020-8-34). The authors have no other conflicts of interest to declare.

Ethical Statement: The authors are accountable for all aspects of the work in ensuring that questions related to the accuracy or integrity of any part of the work are appropriately investigated and resolved. All procedures performed in this study involving human participants will be in accordance with the Declaration of Helsinki (as revised in 2013). This study was approved by the Institution Review Board of the China-Japan Friendship Hospital (Approval number:2020-89-K53). Informed consent will be obtained from each patient before taking part.

Open Access Statement: This is an Open Access article distributed in accordance with the Creative Commons Attribution-NonCommercial-NoDerivs 4.0 International License (CC BY-NC-ND 4.0), which permits the noncommercial replication and distribution of the article with the strict proviso that no changes or edits are made and the original work is properly cited (including links to both the formal publication through the relevant DOI and the license). See: https://creativecommons.org/licenses/by-nc-nd/4.0/.

\section{References}

1. Ratto C, Grillo E, Parello A, et al. Endoanal ultrasoundguided surgery for anal fistula. Endoscopy 2005;37:722-8.

2. Limura E, Giordano P. Modern management of anal fistula. World J Gastroenterol 2015;21:12-20.

3. Mei Z, Wang Q, Zhang Y, et al. Risk Factors for Recurrence after anal fistula surgery: A meta-analysis. Int J Surg 2019;69:153-64.

4. Amato A, Bottini C, De Nardi P, et al. Evaluation and management of perianal abscess and anal fistula: SICCR position statement. Tech Coloproctol 2020;24:127-43. 
5. Sneider EB, Maykel JA. Anal abscess and fistula. Gastroenterol Clin North Am 2013;42:773-84.

6. Zubaidi AM. Anal fistula. Past and present. Saudi Med J 2014;35:937-44.

7. Cadeddu F, Salis F, Lisi G, et al. Complex anal fistula remains a challenge for colorectal surgeon. Int J Colorectal Dis 2015;30:595-603.

8. Pigot F. Treatment of anal fistula and abscess. J Visc Surg 2015;152:S23-9.

9. Wilhelm A. Anal fistula surgery: what are the patients expecting from the surgeon? Tech Coloproctol 2017;21:491-2.

10. Dziki A, Bartos M. Seton treatment of anal fistula: experience with a new modification. Eur J Surg 1998;164:543-8.

11. Hämäläinen KP, Sainio AP. Cutting seton for anal fistulas: high risk of minor control defects. Dis Colon Rectum 1997;40:1443-6; discussion 1447.

12. García-Aguilar J, Belmonte C, Wong DW, et al. Cutting seton versus two-stage seton fistulotomy in the surgical management of high anal fistula. Br J Surg 1998;85:243-5.

13. Vogel JD, Johnson EK, Morris AM, et al. Clinical Practice Guideline for the Management of Anorectal Abscess, Fistula-in-Ano, and Rectovaginal Fistula. Dis Colon Rectum 2016;59:1117-33.

14. Wanitsuwan W, Junmitsakul K, Jearanai S, et al. VideoAssisted Ligation of Intersphincteric Fistula Tract for Complex Anal Fistula: Technique and Preliminary Outcomes. Dis Colon Rectum 2020;63:1534-40.

15. Tao Y, Han JG, Wang ZJ, et al. Long-term efficacy of bioprosthetic anal fistula plug in the treatment of transsphincteric anal fistula. Zhonghua Wei Chang Wai Ke Za Zhi 2020;23:71-5.

16. Hong KD, Kang S, Kalaskar S, et al. Ligation of intersphincteric fistula tract (LIFT) to treat anal fistula: systematic review and meta-analysis. Tech Coloproctol 2014;18:685-91.

17. Bance RR, Coyle P, Dias A, et al. Reducing the rate of fistula: does a fibrin sealant act as an adjunct in pharyngeal closure? A two-centre experience. J Laryngol Otol 2020;134:623-5.

18. Shirah BH, Shirah HA. The Impact of the Outcome of Treating a High Anal Fistula by Using a Cutting Seton and Staged Fistulotomy on Saudi Arabian Patients. Ann Coloproctol 2018;34:234-40.

19. Jamshidi R, Schecter WP. Biological dressings for the management of enteric fistulas in the open abdomen: a preliminary report. Arch Surg 2007;142:793-6.

20. Ritchie RD, Sackier JM, Hodde JP. Incontinence rates after cutting seton treatment for anal fistula. Colorectal Dis 2009;11:564-71.

21. Isbister $\mathrm{WH}, \mathrm{Al}$ Sanea $\mathrm{N}$. The cutting seton: an experience at King Faisal Specialist Hospital. Dis Colon Rectum 2001;44:722-7.

22. Erol T, Mentes B, Bayri H, et al. Preventing the recurrence of acute anorectal abscesses utilizing a loose seton: a pilot study. Pan Afr Med J 2020;35:18.

23. Kelly ME, Heneghan HM, McDermott FD, et al. The role of loose seton in the management of anal fistula: a multicenter study of 200 patients. Tech Coloproctol 2014;18:915-9.

24. Kristo I, Stift A, Staud C, et al. The type of loose seton for complex anal fistula is essential to improve perianal comfort and quality of life. Colorectal Dis 2016;18:O194-8.

25. Zheng L, Shi Y, Zhi C, et al. Loose combined cutting seton for patients with high intersphincteric fistula: a retrospective study. Ann Transl Med 2020;8:1236.

26. Chan AW, Tetzlaff JM, Altman DG, et al. SPIRIT 2013 statement: defining standard protocol items for clinical trials. Ann Intern Med 2013;158:200-7.

27. Durgun V, Perek A, Kapan M, et al. Partial fistulotomy and modified cutting seton procedure in the treatment of high extrasphincteric perianal fistulae. Dig Surg 2002;19:56-8.

28. Westerterp M, Volkers NA, Poolman RW, et al. Anal fistulotomy between Skylla and Charybdis. Colorectal Dis 2003;5:549-51.

(English Language Editor: J. Teoh)
Cite this article as: Cheng Y, Zheng L, Shi Y, Zhi C, Shan J, Sun Y, Guo H, Liu D, Zhang Y. Loose combined cutting seton for patients with suprasphincteric anal fistula: a randomized clinical trial protocol. Ann Palliat Med 2021;10(9):10022-10030. doi: 10.21037/apm-21-2303 